\title{
RECENT ADVANCES IN UNDERSTANDING THE LYMPHATIC AND GLYMPHATIC SYSTEMS OF THE BRAIN
}

\author{
Marian Simka \\ Department of Nursing, College of Applied Sciences, Ruda Śląska, Poland
}

EDITORIAL REVIEW

Phlebological Review 2015; 23, 3: 69-71

DOI: 10.5114/pr.2015.57465

Submitted: 21.11.2015

Accepted: 21.11.2015

\section{ADDRESS FOR CORRESPONDENCE \\ Marian Simka \\ Department of Nursing College of Applied Sciences \\ Królowej Jadwigi 18, 41-704 Ruda Śląska \\ e-mail: mariansimka@poczta.onet.pl}

\begin{abstract} the structure composed of genuine lymphatic vessels. The other, so-called glymphatic brain is likely to play a substantial role in the pathogenesis of many neuroimmune and neurodegenerative disorders, such as: multiple sclerosis, Parkinson's disease, and
\end{abstract}

Key words: brain, lymphatic vessels, glymphatic system.
It this editorial review I will summarise current knowledge on the lymphatic system of the brain. It has long been believed that the central nervous system is characterised by the lack of a lymphatic system and that the role of lymphatic system is played by cerebrospinal fluid. This paradigm was supported by the fact that anatomical structures typical for lymphatic vessels were not found on dissections of the brain. On the other hand, it was known that metabolically active organs are typically characterised by well developed lymphatics. Thus, the fact that the most metabolically active organ seemed to be deprived of lymphatic vessels seemed strange. Also, the anatomy of the brain, namely the fact that the distances between interstitial cerebral fluid and cerebrospinal fluid contained in the cerebral ventricles and subarachnoid space appear to be too great to enable efficient exchange of the soluble, tells us that cerebrospinal fluid by itself cannot play the role of a lymphatic system. Although in the 1980s some researchers put forward the idea that removal of waste products from the brain may take place in the perivascular spaces, where exchange between cerebrospinal fluid and interstitial cerebral fluid is possible, an exact mechanism of this hypothetical process remained elusive.

During last few years part of this great enigma has finally been resolved and now we know that not only is the brain equipped with its own unique lymphatic system, but also that this system consists of two anatomically and functionally very distinct parts. One part of this system is a "classic" lymphatic system, i.e. a structure composed of genuine lymphatic vessels. The other, so-called glymphatic system, is primarily built by astrocytes. It should be emphasised that the majority of currently available knowledge on lymphatics of the central nervous system comes from research on mice and other rodents, while the anatomy and physiology of this newly discovered system in humans - although it is likely to be structured similarly to that of laboratory animals - remains largely unknown (a preliminary study on cadaveric human dura mater revealed the presence of lymphatic vessels similar to those of the rodents) [1]. Respecting these caveats, it seems safe to say that probably many basic assumptions regarding the aetiology of many neuroimmune and neurodegenerative disorders (such as: multiple sclerosis, and Parkinson's and Alzheimer's diseases) and also of traumatic brain injury may need reassessment, since the newly discovered lymphatic system may play a substantial role here [2].

\section{"CLASSIC" LYMPHATIC SYSTEM OF THE BRAIN}

Recent research performed on mice revealed the presence of lymphatic vessels running parallel to the cerebral sinuses (the transverse and superior sagittal sinuses, but probably also other sinuses of the dura mater) [1]. Further evaluation of these structures confirmed that they were not blood vessels: these vessels did not fill with fluorescent dye that had been injected intravenously, they were equipped with classic markers of lymphatic endothelium [Prox1, chemokine CCL21, lymphatic vessel endothelial hyaluronan receptor 1 (Lyve-1), vascular endothelial growth factor receptor-3 (VEGFR3), and a punctuate expression of VE-cadherin] and they contained leuko- 
cytes but not erythrocytes. Interestingly, the endothelial layer of these meningeal lymphatic vessels was not surrounded by smooth muscle cells, and electron microscopy demonstrated non-continuous basement membrane (this finding was in line with punctuate expression of VE-cadherin). Also, expression of integrin- $\alpha 9$, which is a marker of lymphatic valves, was not found. Thus, lymphatic vessels of the brain are structured typically for initial lymphatics, while, as yet, collecting lymphatics have not been found in this organ. These lymphatic vessels were functionally connected with both cerebrospinal fluid and the systemic lymphatic system: a dye injected into cisterna magna of the brain was found in the cerebral lymphatic vessels after 30 minutes and was later demonstrated in the deep cervical lymph nodes [1]. All these findings suggest that the brain is indeed equipped with lymphatic vessels that enable the drainage of waste products and cells of the immune system from cerebrospinal fluid to the lymph nodes (primarily: the deep cervical ones).

\section{THE GLYMPHATIC SYSTEM}

The discovery of the glymphatic system of the brain was inspired by an observation that cerebrospinal fluid can flux deep into the brain parenchyma along the perivascular spaces surrounding the penetrating cerebral arteries. Here cerebrospinal fluid can exchange soluble particles with the interstitial fluid. It seems that arterial pulsation is an important force driving this perivascular flow of cerebrospinal fluid. Further research has revealed that a net flow of water in periarterial space is not directed from the interstitial space toward cerebrospinal fluid, as might be expected in the case of passive diffusion, but rather - interstitial fluid is cleared through the perivascular spaces surrounding draining veins. It has also been demonstrated that this is an active process executed by astrocytes through aquaporin- 4 water channels $[3,4]$. Since glial cells (astrocytes) seem to be the main actor in this process, the researchers coined the term "glymphatic" system to emphasise the role of glial cells playing the role of lymphatics (Fig. 1). Thus, the glymphatic system, thanks to aquaporin- 4 water channels, enables the convective flow of interstitial fluid from the periarterial to the perivenous space. This system allows clearance of the brain from waste products, including substances that are suspected to play a pivotal role in the pathogenesis of neurodegenerative diseases (such as: $\beta$-amyloid in Alzheimer's disease). In addition, the glymphatic system is probably responsible for spreading within brain parenchyma such compounds as: glucose, amino acids, neuromodulators, and growth factors [5, 6]. Interestingly, the glymphatic system is mainly active during sleep [7], and - to further add to the confusion - the study on rats revealed that it is more active during sleep in the lateral and supine positions (although the research in this area is still in its infancy, it is likely that the most popular lateral sleep posture in humans has evolved to optimise the function of the glymphatic system in the sleeping brain [8]). Although functional and anatomic connections between lymphatic and glymphatic parts of the cerebral drainage system have not yet been deciphered, probably waste products drained by the glymphatic system collect in the perivenous space, from which they enter meningeal lymphatic vessels and through the cervical lymph node are transported to the general circulation [9].

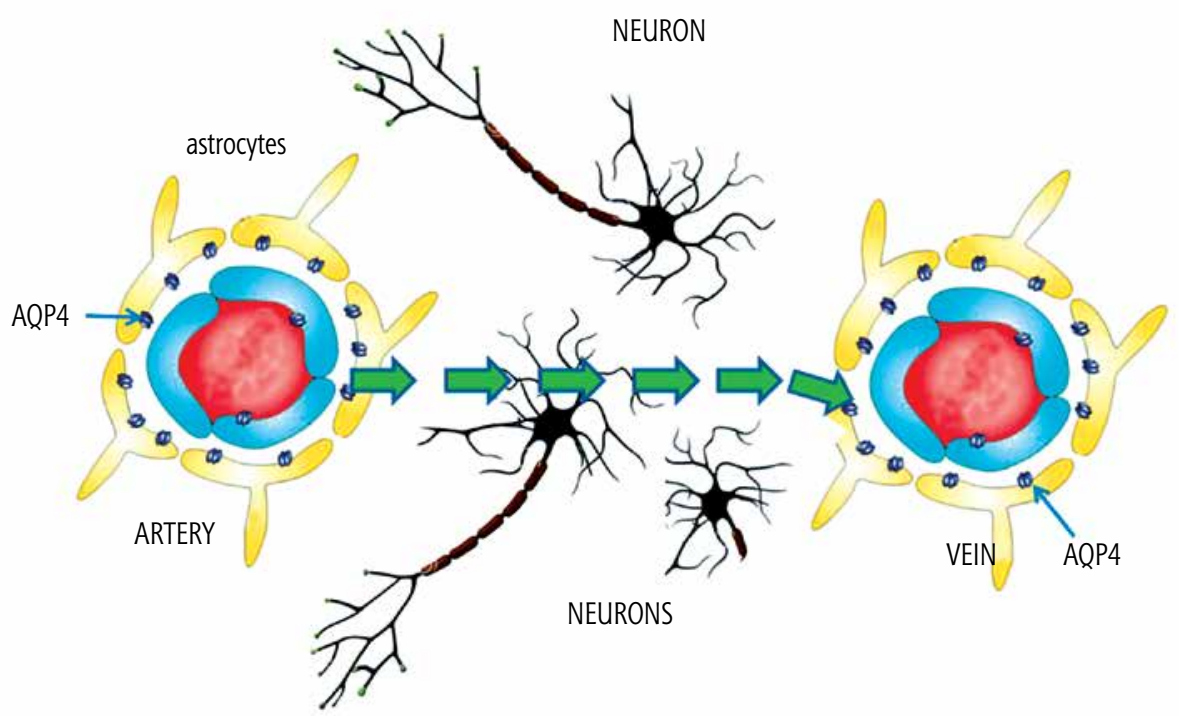

Fig. 1. Glymphatic system of the brain: convective flow of interstitial fluid from the periarterial to the perivenous space enabled by aquaporin-4 water channels of astrocytes (AQP4 - aquaporin-4) 


\section{IMPLICATIONS OF THE DISCOVERY FOR NEUROLOGICAL DISEASE}

The glymphatic system may play an important role in a number of neurodegenerative diseases: Alzheimer's disease, Parkinson's disease, Huntington's disease, and amyotrophic lateral sclerosis. All these pathologies share a common pathomechanism: an accumulation of misfolded, prion-like proteins in the neurons [10-12]. It is tempting to speculate that impaired function of the meningeal lymphatic vessels and also venous congestion within cerebral venous microvasculature (that probably accompanies so-called chronic cerebrospinal venous insufficiency) may impede clearance of pathological proteins and in such a way may promote neurodegenerative diseases $[13,14]$. Indeed, at least some neurodegenerative diseases are more common in the settings of chronic cerebrospinal venous insufficiency [14-17]. By the same token, abnormal function of cerebral lymphatic and glymphatic systems can be responsible for the development of such a neuroinflammatory disease as multiple sclerosis.

The author declares no conflict of interest.

\section{References}

1. Louveau A., Smirnov I., Keyes T.J., Eccles J.D., Rouhani S.J., Peske J.D., Derecki N.C., Castle D., Mandell J.W., Lee K.S., Harris T.H., Kipnis J. Structural and functional features of central nervous system lymphatic vessels. Nature 2015; 523: 337-341.

2. Dissing-Olesen L., Hong S., Stevens B. New brain lymphatic vessels drain old concepts. EBioMedicine 2015; 2: 776-777.

3. Iliff J.J., Nedergaard M. Is there a cerebral lymphatic system? Stroke 2013; 44 (6 Suppl 1): S93-S95.

4. Yang L., Kress B.T., Weber H.J., Thiyagarajan M., Wang B., Deane R., Benveniste H., Iliff J.J., Nedergaard M. Evaluating glymphatic pathway function utilizing clinically relevant intrathecal infusion of CSF tracer. J Transl Med 2013; 11: 107.

5. Iliff J.J., Wang M., Liao Y., Plogg B.A., Peng W., Gundersen G.A., Benveniste H., Vates G.E., Deane R., Goldman S.A., Nagelhus E.A., Nedergaard M. A paravascular pathway facilitates CSF flow through the brain parenchyma and the clearance of interstitial solutes, including amyloid. Sci Transl Med 2012; 4: $147 \mathrm{ra} 111$.

6. Iliff J.J., Lee H., Yu M., Feng T., Logan J., Nedergaard M., Benveniste $\mathrm{H}$. Brain-wide pathway for waste clearance captured by contrast-enhanced MRI. J Clin Invest 2013; 123: 1299-1309.

7. Xie L., Kang H., Xu Q., Chen M.J., Liao Y., Thiyagarajan M., O’Donnell J., Christensen D.J., Nicholson C., Iliff J.J., Takano T., Deane R., Nedergaard M. Sleep drives metabolite clearance from the adult brain. Science 2013; 342: 373-377.

8. Lee H., Xie L., Yu M., Kang H., Feng T., Deane R., Logan J., Nedergaard M., Benveniste $\mathrm{H}$. The effect of body posture on brain glymphatic transport. J Neurosci 2015; 35: 11034-11044.

9. Iliff J.J., Goldman S.A., Nedergaard M. Implications of the discovery of brain lymphatic pathways. Lancet Neurol 2015; 14: 977-979.
10. Dunning C.J., George S., Brundin P. What's like about the prion-like hypothesis for the spreading of aggregated a-synuclein in Parkinson's disease? Prion 2013; 7: 92-97.

11. McLennan N.F., Brennan P.M., McNeill A., Davies I., Fotheringham A., Rennison K.A., Ritchie D., Brannan F., Head M.W., Ironside J.W., Williams A., Bell J.E. Prion protein accumulation and neuroprotection in hypoxic brain damage. Am J Pathol 2004; 165: 227-235.

12. Visanji N.P., Brooks P.L., Hazrati L.N., Lang A.E. The prion hypothesis in Parkinson's disease: Braak to the future. Acta Neuropathol 2013; 1: 2 .

13. Gallina P., Scollato A., Conti R., Di Lorenzo N., Porfirio B. A $\beta$ Clearance, "hub" of multiple deficiencies leading to Alzheimer disease. Front Aging Neurosci 2015; 7: 200.

14. Zivadinov R. Is there a link between the extracranial venous system and central nervous system pathology? BMC Med 2013; 11: 259 .

15. Beggs C., Chung C.P., Bergsland N., Wang P.N., Shepherd S., Cheng C.Y., Dwyer M.G., Hu H.H., Zivadinov R. Jugular venous reflux and brain parenchyma volumes in elderly patients with mild cognitive impairment and Alzheimer's disease. BMC Neurol 2013; 13: 157.

16. Chung C.P., Beggs C., Wang P.N., Bergsland N., Shepherd S., Cheng C.Y., Ramasamy D.P., Dwyer M.G., Hu H.H., Zivadinov R. Jugular venous reflux and white matter abnormalities in Alzheimer's disease: a pilot study. J Alzheimers Dis 2014; 39: 601-609.

17. Simka M. What is the relationship between chronic cerebrospinal venous insufficiency and multiple sclerosis? Rev Vasc Med 2013; 1: 66-70. 